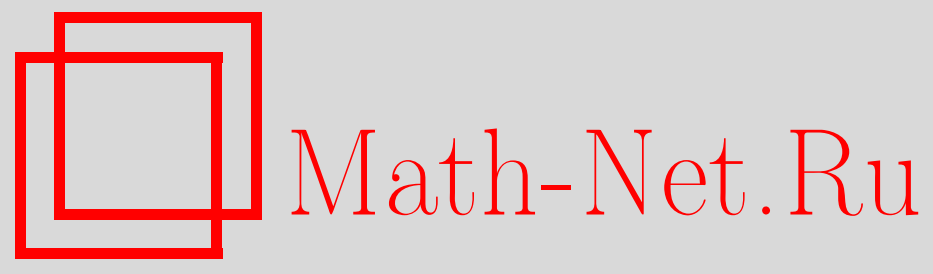

А. В. Лебедев, Максимумы независимых сумм в случае тяжелых хвостов, Теория вероятн. и ее примен., 2004, том 49, выпуск 4, 791-794

DOI: https://doi.org/10.4213/tvp196

Использование Общероссийского математического портала Math-Net.Ru подразумевает, что вы прочитали и согласны с пользовательским соглашением

http: //www . mathnet.ru/rus/agreement

Параметры загрузки:

IP : 18.234 .156 .22

26 апреля 2023 г., 11:44:50

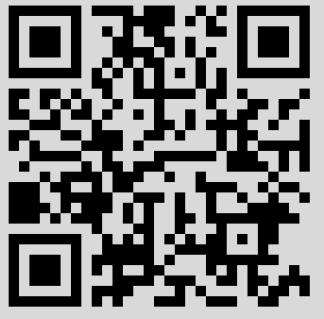


(c) $2004 \Gamma$.

ЛЕБЕДЕВ А. В.*

\section{МАКСИМУМЫ НЕЗАВИСИМЫХ СУММ В СЛУЧАЕ ТЯЖЕЛЫХ ХВОСТОВ ${ }^{1)}$}

Рассматривается семейство экстремумов вида

$$
Y_{m n}=\max _{1 \leqslant i \leqslant m} \sum_{j=1}^{n} X_{i j}, \quad m, n \geqslant 1,
$$

где $X_{i j}, i, j \geqslant 1$, независимы и имеют одинаковое распределение $F$, обладаюшее свойством субэкспоненциальности. Изучается предельное поведение $Y_{m n}$ при $m, n \rightarrow \infty$. Получены различные невырожденные предельные законы (Фреше и Гумбеля) в зависимости от характера относительного роста $m, n$ и свойств хвостов $F$.

Ключевые слова и фразы: максимумы, суммы, правильно меняющиеся хвосты, субэкспоненциальность, невырожденные предельные законы, линейная нормировка.

Рассмотрим семейство экстремумов вида

$$
Y_{m n}=\max _{1 \leqslant i \leqslant m} \sum_{j=1}^{n} X_{i j}, \quad m, n \geqslant 1,
$$

где $X_{i j}, i, j \geqslant 1$, независимы и имеют одинаковое распределение $F$. Нас интересует предельное поведение $Y_{m n}$ при $m, n \rightarrow \infty$.

Подобные вопросы изучались еше в [1]. Некоторые новые результаты были получены в [2].

Выражение (1) может описывать, например, время выполнения однотипных работ, проводимых параллельно, если каждая из них делится на множество фаз [3]. Особый интерес здесь представляет модель параллельных вычислений на компьютере с большим числом процессоров. В [2] упоминались некоторые нормы матриц из независимых случайных элементов. Формулу (1) можно также рассматривать как дискретизацию (по времени) максимума $m$ случайных процессов с независимыми прирашениями. Подобная модель для гауссовских процессов с непрерывным временем изучалась в [4].

Основные результаты [2] получены при условии, что $F$ обладает конечными средним и дисперсией. В этих предположениях суммы в (1) оказываются асимптотически нормальными, а для максимумов независимых нормальных случайных величин имеет место предельный закон Гумбеля. Таким образом, при $\mathbf{E} X_{i j}=0, \mathbf{D} X_{i j}=1$ и некоторых ограничениях на относительный рост $m, n \rightarrow \infty$ были получены пределы вида

$$
\mathbf{P}\left\{a_{m} n^{-1 / 2}\left(Y_{m n}-b_{m}\right) \leqslant x\right\} \rightarrow \exp \left\{-e^{-x}\right\}
$$

где $a_{m}=(2 \ln m)^{1 / 2}, b_{m}=(2 \ln m)^{1 / 2}-(2 \ln m)^{-1 / 2} \ln (4 \pi \ln m)^{1 / 2}$.

Далее мы изучим случай распределений $F$ с тяжелыми хвостами, не обязательно имеющих конечные средние и дисперсии, но зато обладающих свойством субэкспоненциальности (см. [5], [6]):

$$
\bar{F}^{* n}(u) \sim n \bar{F}(u), \quad u \rightarrow \infty, \quad n \geqslant 2, \quad \text { где } \bar{F}(u)=1-F(u) .
$$

* Московский государственный университет им. М.В. Ломоносова, механикоматематический факультет, кафедра теории вероятностей, Ленинские горы, 119992 Москва, Россия; e-mail: alebedev@mech.math.msu.su

1) Работа выполнена при поддержке грантов РФФИ 03-01-00724, 04-01-00700 и гранта НШ 1758.2003.1. 
Свойство (3) может иметь место и при $n \rightarrow \infty$, если наложить определенные условия на рост $u$. Фактически, речь идет о больших уклонениях при нарушении условия Крамера.

Рассмотрим сначала случай, когда $F$ имеет правильно меняющийся хвост, т.е. $\bar{F}(u) \sim u^{-\alpha} L(x), u \rightarrow \infty$, где $\alpha>0$ и $L(x)$ - медленно меняющаяся функция [7], [8]. Введем последовательность чисел $c_{n}$ таких, что $n \bar{F}\left(c_{n}\right) \rightarrow 1, n \rightarrow \infty$. Тогда $c_{n} \sim n^{1 / \alpha} L^{*}(n), n \rightarrow \infty$, где $L^{*}(x)$ - медленно меняюшаяся функция, зависящая oт $L$ (cм. [8]).

Заметим, что если асимптотическое соотношение (3) выполняется при $u_{m, n}=$ $c_{m n} x, x>0$, то из него следует

$$
\mathbf{P}\left\{c_{m n}^{-1} Y_{m n} \leqslant x\right\} \longrightarrow \exp \left\{-x^{-\alpha}\right\}, \quad x>0,
$$

т.е. имеет место предельный закон Фреше с тем же показателем. Здесь используем тот факт, что если $k \bar{F}\left(u_{k}\right) \rightarrow \tau, k \rightarrow \infty$, то $F^{k}\left(u_{k}\right) \rightarrow e^{-\tau}$.

Далее будем предполагать для простоты, что $\mathbf{E} X_{i j}=0$ при $\alpha>1$ и $\mathbf{D} X_{i j}=1$ при $\alpha>2$ (в противном случае легко произвести линейную перенормировку).

Теорема 1. Если $\alpha \in(0,1) \cup(1,2) u F(-x) / \bar{F}(x) \rightarrow r \geqslant 0, x \rightarrow \infty$, mo npu $m, n \rightarrow \infty$ верно (4).

Д о к а за т е л ь с т в о. При условиях теоремы $F$ принадлежит области притяжения устойчивого закона с показателем $\alpha$ (см. [7]), и, согласно [9], соотношение (3) выполняется при $u / c_{n} \rightarrow \infty$. Для $u_{m, n}=c_{m n} x, x>0$, это условие выполняется, так как $c_{m n} / c_{n} \rightarrow \infty$ при $m, n \rightarrow \infty$.

Таким образом, в данном случае никаких ограничений на относительный рост $m, n \rightarrow \infty$ нет.

Случай $\alpha=1$, по-видимому, требует более тонкого анализа, однако (4) выполняется, например, для распределения Коши, что было отмечено в [2].

Теорема 2. Пусть $\alpha>2 u \mathbf{E}\left|X_{i j}\right|^{2+\delta}<\infty$ для некоторого $\delta>0$, тогда:

1) если $\limsup \sup _{m, n \rightarrow \infty}(\ln m / \ln n)<\alpha / 2-1$, то верно (2);

2) если $\liminf \operatorname{in}_{m \rightarrow \infty}(\ln m / \ln n)>\alpha / 2-1$, то верно (4).

Д ок а з а тель с т в о. При данных условиях по теореме 1.9 из [10] имеет место асимптотическое соотношение

$$
\bar{F}^{* n}(u)=\bar{\Phi}\left(n^{-1 / 2} u\right)(1+o(1))+n \bar{F}(u)(1+o(1)), \quad u \geqslant n^{1 / 2},
$$

где $\Phi(x)$ - функция стандартного нормального распределения.

Пусть выполнено условие в 1). Положим $u_{m, n}=n^{1 / 2}\left(b_{m}+x / a_{m}\right)$, тогда $u_{m, n} \sim$ $(2 n \ln m)^{1 / 2}$, и $n \bar{F}\left(u_{m, n}\right)=o\left(\bar{\Phi}\left(u_{m, n} / n^{1 / 2}\right)\right), m, n \rightarrow \infty$. При этом условии из (5) следует (2).

Пусть выполнено условие в 2). Положим $u_{m, n}=c_{m n} x, x>0$, тогда $u_{m, n} \sim$ $x(m n)^{1 / \alpha} L^{*}(m n)$, и $\bar{\Phi}\left(u_{m, n} / n^{1 / 2}\right)=o\left(n \bar{F}\left(u_{m, n}\right)\right), m, n \rightarrow \infty$. При этом условии из $(5)$ получаем (3), откуда следует (4).

3 а м е ч а н и е 1 . Условие $\mathbf{E}\left|X_{i j}\right|^{2+\delta}<\infty$ для некоторого $\delta>0$ заведомо выполняется при $\alpha>2$, если $F(-x) / \bar{F}(x) \rightarrow r \geqslant 0, x \rightarrow \infty$. Последнее верно, например, когда случайные величины ограничены снизу $(r=0)$, что имеет место, если мы рассматриваем времена, как в [3] (нормированные средним и дисперсией).

3 а м е ч а н и е 2. В случае степенных хвостов условие 2) удается ослабить до $m /\left\{n^{\alpha / 2-1}(\ln n)^{\alpha / 2}\right\} \rightarrow \infty$.

3 а м е ч а н и е 3 . Теорема 1 обеспечивает устойчивость сходимости: в любом случае имеет место сходимость к одному предельному закону с единой линейной нормировкой. Теорема 2, напротив, показывает неустойчивость: в зависимости от характера относительного роста $m, n \rightarrow \infty$ возможна сходимость к двум различным предельным законам (при соответствуюших различных нормировках).

Как известно еще из [5], класс субэкспоненциальных распределений помимо распределений с правильно меняющимися хвостами включает в себя и некоторые распределения, чьи хвосты убывают быстрее любой степени (хотя и медленнее экспоненты, что дало название всему классу), в частности, распределение Вейбулла с показателем меньше единицы. 
Рассмотрим случай распределений с «вейбулловскими» хвостами:

$$
\bar{F}(x) \sim \exp \left\{-\left(\frac{x}{c}\right)^{\beta}\right\}, \quad x \rightarrow \infty, \quad 0<\beta<1, \quad c>0 .
$$

Для таких распределений имеет место предел

$$
\bar{F}\left(b_{k}^{*}+\frac{x}{a_{k}^{*}}\right) \rightarrow e^{-x}, \quad k \rightarrow \infty
$$

где $a_{k}^{*}=\beta(\ln k)^{1-1 / \beta} / c, b_{k}^{*}=c(\ln k)^{1 / \beta}, k>1$.

Если для $u_{m, n}=b_{m n}^{*}+x / a_{m n}^{*}$ окажется выполненным (3), то из (7) будет следовать

$$
\mathbf{P}\left\{a_{m n}^{*}\left(Y_{m n}-b_{m n}^{*}\right) \leqslant x\right\} \rightarrow \exp \left\{-e^{-x}\right\} .
$$

Как отмечено в [10], достаточное условие выполнения (3) в этом случае имеет вид $u>\rho(n) n^{1 /(2(1-\beta))}$, где $\rho(n)$ - некоторая функция, медленно стремяшаяся к бесконечности при $n \rightarrow \infty$.

Теорема 3. Если выполнено (6) и

$$
\liminf _{m, n \rightarrow \infty} \frac{\ln \ln m}{\ln n}>\frac{\beta}{2(1-\beta)}
$$

то верно (8).

Д ок а 3 а т ел ь с т в о. Из условия теоремы следует сушествование $\varepsilon>0$ такого, что при всех достаточно больших $m, n$ верно $\ln m>n^{(1+\varepsilon) \beta /(2(1-\beta))}$. Положим $u_{m, n}=b_{m n}^{*}+x / a_{m n}^{*}$, тогда $u_{m, n} \sim c(\ln m)^{1 / \beta}$, поскольку $\ln n=o(\ln m)$, и при всех достаточно больших $m$ верно $u_{m, n}>p(\ln m)^{1 / \beta}$, где $0<p<c$. Отсюда при всех достаточно больших $m, n$ получаем $u_{m, n}>p n^{(1+\varepsilon) /(2(1-\beta))}>\rho(n) n^{1 /(2(1-\beta))}$, что и доказывает теорему.

3 а м е ч а н и е 4. Распределения типа (6), если их левые хвосты также убывают быстрее любой степени, имеют моменты любого порядка, так что по теореме 1 из [2] для них верно (2) при $m=O\left(n^{s}\right)$ с любым $s<\infty$. Ситуация в каком-то смысле обратная той, что в теореме 2: предельный закон здесь один (Гумбеля), но при различном характере относительного роста $m, n \rightarrow \infty$ требуется различная линейная нормировка. Вопрос о том, что происходит в промежуточной зоне, остается открытым для дальнейших исследований.

\section{СПИСОК ЛИТЕРАТУРЫ}

1. Ивченко Г.И. Вариационный ряд для схемы суммирования независимых величин. - Теория вероятн. и ее примен., 1973 , т. 18 , в. 3 , с. $557-570$.

2. Лебедев $A . B$. Предельные теоремы для максимумов независимых случайных сумм. - Теория вероятн. и ее примен., 1999, т. 44, в. 3, с. 631-634.

3. Kang S., Serfozo R. F. Extreme values of phase-type and mixed random variables with parallel-processing examples. - J. Appl. Probab., 1999, v. 36, № 1, p. 194-210.

4. Мачак И.К. Об относительной устойчивости экстремальных случайных функций. - Матем. заметки, 2002, т. 71, № 5, с. 787-790.

5. Чистяков В. П. Теорема о суммах независимых положительных случайных величин и ее приложения к ветвящимся случайным процессам. - Теория вероятн. и ее примен., 1964 , т. 9 , в. 4, с. $710-718$.

6. Рогозин Б. А. О постоянной в определении субэкспоненциальных распределений. - Теория вероятн. и ее примен., 1999, т. 44, в. 2, с. 455-458.

7. Феллер В. Введение в теорию вероятностей и ее приложения. Т. 2. М.: Мир, 1984, $752 \mathrm{c}$.

8. Сенета Е. Правильно меняющиеся функции. М.: Наука, 1985, 142 с. 
9. Ткачук С. Г. Теорема о больших уклонениях в $\mathbf{R}^{s}$ в случае устойчивого предельного закона. - Случайные процессы и статистические выводы. Ташкент: Фан, 1974 , в. 4 , c. $178-184$.

10. Nagaev $S . V$. Large deviations of sums of independent random variables. - Ann. Probab., 1979, v. 7, № 5, p. 745-789.

Поступила в редакцию 17.III.2003

(c) $2004 \mathrm{r}$.

HAГAEB C. B.*

\section{О БОЛЬШИХ УКЛОНЕНИЯХ АВТОНОРМИРОВАННОЙ СУММЫ ${ }^{1)}$}

В работе выведены экспоненциальные оценки для вероятностей больших уклонений автонормированной суммы независимых случайных величин. При этом не предполагается, что слагаемые одинаково распределены.

Ключевые слова и Фразы: неравенство Берри-Эссеена, отношение Ляпунова, автонормированная сумма, большие уклонения.

1. Введение. Пусть $X_{1}, \ldots, X_{n}$ - независимые случайные величины, $S_{n}=$ $\sum_{i=1}^{n} X_{i}$. Обозначим $b_{j}^{2}=\mathbf{E} X_{j}^{2}, B_{n}^{2}=\sum_{j=1}^{n} b_{j}^{2}, \beta_{3, j}=\mathbf{E}\left|X_{j}\right|^{3}, C_{n}=\sum_{j=1}^{n} \beta_{3, j}$, $L_{n}=C_{n} / B_{n}^{3}$.

Положим $V_{n}^{2}=\sum_{i=1}^{n} X_{i}^{2}$. Целью настояшей работы является изучение больших уклонений автонормированной суммы $S_{n} / V_{n}$, точнее вывод верхних оценок для вероятности $\mathbf{P}\left\{S_{n}>V_{n} x\right\}$ с явно вычисленными постоянными.

Автонормированные суммы изучаются уже давно (см., например, [1]), однако в основном с асимптотической точки зрения и для одинаково распределенных слагаемых (подробную библиографию см., например, в [2]). К.-М. Шао [3] показал, что если $X_{1}, \ldots, X_{n}$ - независимые одинаково распределенные случайные величины с нулевым средним и конечной дисперсией, то для любого $2 \leqslant x \leqslant n^{1 / 2} / 2$

$$
\mathbf{P}\left\{S_{n}>x V_{n}\right\} \leqslant\left(1+x^{-1}\right) \frac{1}{\sqrt{2 \pi} x} \exp \left(-\frac{x^{2}}{2}+A x^{2} \Delta_{n, x}\right),
$$

где $A-$ абсолютная постоянная,

$$
\Delta_{n, x}=\mathbf{E} X^{2} I\left(|X|>\frac{\sqrt{n}}{x}\right)+\frac{x}{\sqrt{n}} \mathbf{E}|X|^{3} I\left(|X| \leqslant \frac{\sqrt{n}}{x}\right) .
$$

Значение постоянной $A$ явно не вычисляется.

К. Ван и Б.-И Джин [4] вывели неравномерную экспоненциальную оценку БерриЭссеена для разности

$$
\delta_{n}(x)=\mathbf{P}\left\{S_{n}<x V_{n}\right\}-\Phi(x),
$$

где $\Phi(x)$ - функция распределения стандартного нормального закона.

С другой стороны, Э. Жине, Ф. Гётце и Д. М. Мейсон [5] доказали, что

$$
\mathbf{E} \exp \left\{\frac{\left(S_{n} / V_{n}\right)^{2}}{4(1+4 e / 3)^{2} M_{n}}\right\} \leqslant 2 \quad \text { и } \quad \mathbf{E} \exp \left\{t\left|\frac{S_{n}}{V_{n}}\right|\right\} \leqslant \exp \left\{\left(1+\frac{4 e}{3}\right) M_{n}^{2} t^{2}\right\}
$$

* Институт математики им. С.Л. Соболева, 630090 Новосибирск-90, Россия; e-mail:nagaev@math.nsk.ru

1) Работа выполнена при поддерже Российского фонда фундаментальных исследований (грант №99-01-00493), а также INTAS (грант № 99-01317). 